\title{
Dynamic characterization of the joints in a beam structure by using spectral element method
}

\author{
Usik Lee \\ Department of Mechanical Engineering, Inha \\ University, 253 Yonghyun-Dong, Nam-Ku, Inchon \\ 402-751, South Korea \\ Fax: +82 32866 1434; E-mail: ulee@inha.ac.kr
}

Received 13 November 2000

Revised 29 September 2001

\begin{abstract}
A frequency-domain approach is introduced to identify the dynamic characteristics of the mechanical joints within a onedimensional structure. In this paper, a cantilevered beam structure with a bolt-joint is considered as an illustrative problem. The beam structure consists of two Timoshenko-beams connected to each other by a bolted joint. The bolt-joint is represented by the equivalent joint model with four parameters, and the four parameters are determined from the experimentally measured frequency response functions (FRF). To improve the accuracy of the identified bolt-joint parameters, the spectral element models are used for two Timoshenkobeams. Experiments are conducted for the cantilevered beam structure and the four bolt-joint parameters determined from the measured FRF. The spectral analysis is conducted to predict the dynamic responses of the beam structure by using the spectral bolt-joint element that is determined from the identified bolt-joint parameters. It is found that the analytical predictions are in general quite in good agreement with the experimentally measured dynamic responses.
\end{abstract}

Keywords: Joint, beam, spectral element method, vibration, frequency response function

\section{Introduction}

The identification of structural parameters has the primary objective of deriving a successful mathematical model of a structure, which reproduces the observed behavior of the structure. However, one often experiences the problem that a mathematical model (e.g., finite element model) does not predict the dynamic be- havior of a real structure very well when compared with the measurements. This discrepancy is mostly due to the inaccurate identification of the structural parameters such as the mass, stiffness, and damping properties of a structure. This is why there have been intense studies in the subject of structural system identification.

Dynamic characteristics of a structure with mechanical joints certainly depend on the dynamic characteristics of the joints. Thus, the dynamic characteristics of the joints should be identified accurately before conducting the vibration analysis for the structure. Though there have been a huge number of publications in the subject of the structural system identification (mostly on the identification of the mass, stiffness, damping, and damage properties of a structure), there are relatively few studies on the identification of the dynamic characteristics of mechanical joints. Yuan and $\mathrm{Wu}$ [1] used the dynamic data system methodology to identify the joint structural parameters while the system was represented by a finite element model. Tsai and Chou [2] extracted the joint properties directly from measured FRF of the substructures and the assembled structure, and the least square method is used. Nabari et al. [3] applied the inverse eigensensitivity method to update the finite element model of a joint. In many studies, the finite element models and the measured structure modal parameters are popularly used for the structural system identification (simply, called FEMmodal parameters method).

As the first issue, it is well known that the finite element model usually provides less accurate structural dynamic characteristics, especially at high frequency. However, in contrast to the conventional finite element model, the spectrally formulated finite element model (simply, spectral element model) has been proved to provide very accurate dynamic characteristics of a structure even at high frequency [4-7]. Since the spectral element is formulated from the exact dynamic shape functions that treat the mass distribution exactly, the spectral element method (SEM) is often 


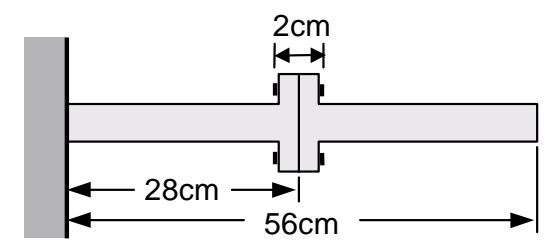

$$
\begin{array}{cl}
\kappa \mathrm{GA}=6.8 \times 10^{6}[\mathrm{~N}], & \mathrm{El}=180\left[\mathrm{~N} \cdot \mathrm{m}^{2}\right] \\
\rho \mathrm{A}=0.84[\mathrm{~kg} / \mathrm{m}], & \rho \mathrm{l}=7 \times 10^{-6}[\mathrm{~N} \cdot \mathrm{m}]
\end{array}
$$

Fig. 1. A cantilevered beam structure with a bolt-joint in the midway.

justifiably referred to as an exact method [7] though its accuracy is still limited by the structure model considered for spectral element formulation. The SEM is a frequency-domain approach in which the forward- and inverse-FFT algorithms are efficiently used. As the second issue, it is not an easy task even today to extract accurate modal parameters from experiments. Thus, as an alternative to the FEM-Modal parameter method, a more reliable and easy-to-use method is desirable.

\section{Problem statement and objectives}

Due to the apparent advantages of SEM, it seems to be reasonable to use the spectral element model for a structure to improve the accuracy of the structural system identification. Since it is not an easy task even today to extract accurate modal parameters from experiments, it seems to be more convenient to directly use the measured FRF in the structural system identification procedure. Hence, this paper introduces a new system identification method based on the SEM and the measured FRF (simply, SEM-FRF approach).

In this paper, a cantilevered beam structure is considered as an application problem. The beam structure consists of two uniform beams and they are connected to each other by the bolt-joint in the midway, as shown in Fig. 1. To apply the SEM technique to both system identification and analytical vibration analysis, the uniform beams are represented by the spectral Timoshenko-beam element models. Consistently, the bolt-joint is also represented by the equivalent spectral element model with four model parameters. Then, this paper introduces a method to determine the bolt-joint model parameters from the experimentally measured FRF. These are detailed in the following sections.

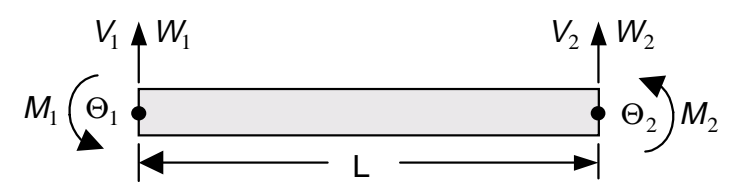

Fig. 2. Sign convention for the spectral Timoshenko-beam element.

\section{Spectral Timoshenko-beam element model}

The spectral element formulation begins with the equations of motion of a structure. For a finite Timoshenko-beam element of length $L$, the equation of motion is given by [8]

$$
\begin{aligned}
& E I \theta^{\prime \prime}+\kappa G A\left(w^{\prime}-\theta\right)-\rho I \ddot{\theta}=0 \\
& \kappa G A\left(w^{\prime \prime}-\theta^{\prime}\right)-\rho A \ddot{w}=0
\end{aligned}
$$

where $w(x, t)$ and $\theta(x, t)$ are the transverse deflection and the angle of rotation due to bending, respectively. $E I$ is the bending rigidity, $\kappa G A$ the shear rigidity, $\rho I$ the mass moment of inertia, and $\rho A$ is the mass density per length of beam. The dynamic responses are assumed in the spectral representation [5] as

$$
\begin{aligned}
& w(x, t)=\sum_{n}^{N} W_{n}\left(x ; \omega_{n}\right) e^{i \omega_{n} t} \\
& \theta(x, t)=\sum_{n}^{N} \Theta_{n}\left(x ; \omega_{n}\right) e^{i \omega_{n} t}
\end{aligned}
$$

The spectral components are spatially dependent Fourier coefficients, and thus they are functions of frequency $\omega$. For shorthand, the summation and the superscripts used for spectral representations will be removed in the following without causing confusions.

For the finite beam element with the (spectral) nodal degrees of freedom (DOF) specified at its ends nodes 1 and 2 (see Fig. 2), the spectral component of the displacement fields can be derived in the form

$$
\boldsymbol{U}(x ; \omega)=\Psi(x ; \omega) \boldsymbol{d}
$$

where

$$
\begin{aligned}
& \boldsymbol{U}(x ; \omega)=\left\{\begin{array}{ll}
W & \Theta
\end{array}\right\}^{T} \\
& \boldsymbol{d}=\left\{W_{1} \Theta_{1} W_{2} \Theta_{2}\right\}^{T}
\end{aligned}
$$

In above Eq. (3), $\Psi(x, t)$ is the frequency-dependent (dynamic) shape functions matrix constructed from the exact solutions of governing differential equations.

The force-displacement relations are given by 


$$
M=E I \Theta^{\prime}
$$

$$
V=-\kappa G A\left(W^{\prime}-\Theta\right)
$$

where $M$ and $V$ are the bending moment and transverse shear force, respectively. By using Eqs (3) and (5), the nodal forces $f$ defined in Fig. 2 can be related to the nodal DOF $\boldsymbol{d}$ as

$$
\boldsymbol{f}=\left\lfloor\boldsymbol{G}(\omega) \boldsymbol{H}^{-1}(\omega)\right\rfloor \boldsymbol{d} \equiv \boldsymbol{S}(\omega) \boldsymbol{d}
$$

where

$$
\begin{aligned}
& \boldsymbol{f}=\left\{\begin{array}{llll}
V_{1} & M_{1} & V_{2} & M_{2}
\end{array}\right\}^{T} \\
& \boldsymbol{G}(\omega)=
\end{aligned}
$$

$$
\left[\begin{array}{cccc}
\eta_{1} & \eta_{2} & -\eta_{1} & -\eta_{2} \\
i \mu_{1} & i \mu_{2} & i \mu_{1} & i \mu_{2} \\
-\eta_{1} \varepsilon_{1}^{-1} & -\eta_{2} \varepsilon_{2}^{-1} & \eta_{1} \varepsilon_{1} & \eta_{2} \varepsilon_{2} \\
-i \mu_{1} \varepsilon_{1}^{-1} & -i \mu_{2} \varepsilon_{2}^{-1} & -i \mu_{1} \varepsilon_{1} & -i \mu_{2} \varepsilon_{2}
\end{array}\right]
$$$$
\boldsymbol{H}(\omega)=\left[\begin{array}{cccc}
1 & 1 & 1 & 1 \\
\alpha_{1} & \alpha_{2} & -\alpha_{1} & -\alpha_{2} \\
\varepsilon_{1}^{-1} & \varepsilon_{2}^{-1} & \varepsilon_{1} & \varepsilon_{2} \\
\alpha_{1} \varepsilon_{1}^{-1} & \alpha_{2} \varepsilon_{2}^{-1} & -\alpha_{1} \varepsilon_{1} & -\alpha_{2} \varepsilon_{2}
\end{array}\right]
$$

$$
\alpha_{n}=-\frac{i b k_{n}}{b-c \omega^{2}+k_{n}^{2}} \quad(n=1,2)
$$$$
\eta_{n}=\kappa G A\left(\alpha_{n}+i k_{n}\right) \quad(n=1,2)
$$

$$
\mu_{n}=E I \alpha_{n} k_{n} \quad(n=1,2)
$$

$$
\varepsilon_{n}=e^{i k_{n} L} \quad(n=1,2)
$$

$$
\begin{aligned}
& k_{1,2}= \\
& \sqrt{\frac{1}{2}\left[(a+c) \omega^{2} \pm \omega \sqrt{4 a b+(a-c)^{2} \omega^{2}}\right]} \\
& a=\frac{\rho A}{\kappa G A}, \quad b=\frac{\kappa G A}{E I}, \quad c=\frac{\rho I}{E I}
\end{aligned}
$$

In Eq. (6), the symmetric matrix $\boldsymbol{S}=\left[s_{i j}\right]$ is the spectral element matrix of Timoshenko-beam. The spectral elements can be assembled to form a global system equation in a completely analogous way to that used for FEM [4].

\section{Four-parameters joint model}

Consider a bolt-joint within a cantilevered beam structure (Fig. 3(a)) as a representative joint model. The bolt-joint is represented by an equivalent model as shown in Fig. 3(b). The equivalent bolt-joint model consists of a (equivalent) lumped mass and a (equivalent) spring-system. The lumped mass has its mass $\mathrm{m}$ and the mass moment of inertia $I$. The spring-system consists of a transverse (extensional) spring $k_{v}$ and a torsional spring $k_{t}$. The lumped mass and the springsystem are connected in series.

By using the concept of four-pole parameters method [8], the transfer matrix for the spring-system can be derived as

$$
\left\{\begin{array}{c}
V_{J} \\
W_{J} \\
M_{J} \\
\Theta_{J}
\end{array}\right\}=\left[\begin{array}{cccc}
1 & 0 & 0 & 0 \\
1 / k_{v} & 1 & 0 & 0 \\
0 & 0 & 1 & 0 \\
0 & 0 & -1 / k_{t} & 1
\end{array}\right]^{-1}\left\{\begin{array}{c}
V_{1} \\
W_{1} \\
M_{1} \\
\Theta_{1}
\end{array}\right\}
$$

where $V_{J}, W_{J}, M_{J}$, and $\Theta_{J}$ are the values at the joint between the lumped mass and the spring-system. Similarly, the transfer matrix for the lumped mass is derived as

$$
\begin{aligned}
\left\{\begin{array}{c}
V_{2} \\
W_{2} \\
M_{2} \\
\Theta_{2}
\end{array}\right\}= & {\left[\begin{array}{cccc}
-1 & -m \omega^{2} & 0 & 0 \\
0 & 1 & 0 & 0 \\
0 & 0 & -1 & -I \omega^{2} \\
0 & 0 & 0 & 1
\end{array}\right]^{-1} } \\
& \left\{\begin{array}{c}
V_{J} \\
W_{J} \\
M_{J} \\
\Theta_{J}
\end{array}\right\}
\end{aligned}
$$

Combining Eqs (8) and (9) gives the transfer matrix which relates the nodal values at one end of bolt-joint (i.e. node 1) to the nodal values at the other end (i.e., node 2):

$$
\begin{aligned}
& \left\{\begin{array}{l}
W_{2} \\
\Theta_{2} \\
V_{2} \\
M_{2}
\end{array}\right\}= \\
& {\left[\begin{array}{cccc}
1 & 0 & -1 / k_{v} & 0 \\
0 & 1 & 0 & 1 / k_{t} \\
-m \omega^{2} & 0 & -1+m \omega^{2} / k_{v} & 0 \\
0 & I \omega^{2} & 0 & -1+I \omega^{2} / k_{t}
\end{array}\right]} \\
& \left\{\begin{array}{c}
W_{1} \\
\Theta_{1} \\
V_{1} \\
M_{1}
\end{array}\right\}
\end{aligned}
$$




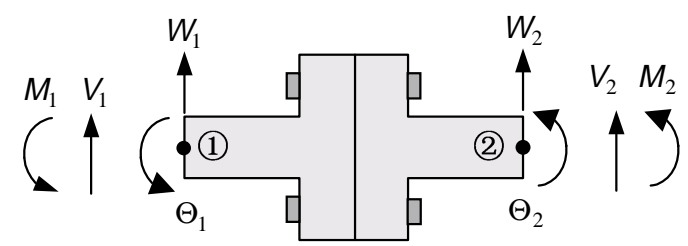

(a) A Bolt-Joint

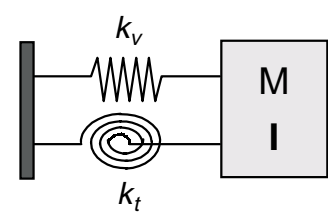

(b) Equivalent Model

Fig. 3. Equivalent bolt-jont model with four parameters.

Note that the sign convention defined in Fig. 3(a), which is different from that commonly used for the transfer matrix [9], is used in Eqs (8)-(10).

By rearranging the nodal values, Eq. (10) can be written into the force-displacement relation as

$$
f=S_{\mathrm{J}}(\omega) \boldsymbol{d}
$$

The same definitions as given in Eqs (4b) and (7a) are consistently used for the vectors $\boldsymbol{f}$ and $\boldsymbol{d}$ in Eq. (11). The symmetric matrix $\boldsymbol{S}_{J}$ is the spectral element matrix for the equivalent bolt-joint model:

$$
\boldsymbol{S}_{J}=\left[\begin{array}{cccc}
k_{v} & 0 & -k_{v} & 0 \\
0 & -k_{t} & 0 & k_{t} \\
-k_{v} & 0 & -m \omega^{2}+k_{v} & 0 \\
0 & k_{t} & 0 & I \omega^{2}-k_{t}
\end{array}\right]
$$

One should note that the spectral element matrix $S_{J}$ includes all of equivalent bolt-joint model parameters. These parameters are to be determined from experimentally measured FRF, which will be discussed in the following section.

\section{Identification of joint parameters}

This section introduces a SEM-based methodology to determine the four parameters of equivalent boltjoint model from the experimentally measured FRF. In SEM, a uniform structural element can be modeled by a single spectral element, regardless of its length, without degrading the solution accuracy at all [4]. However,

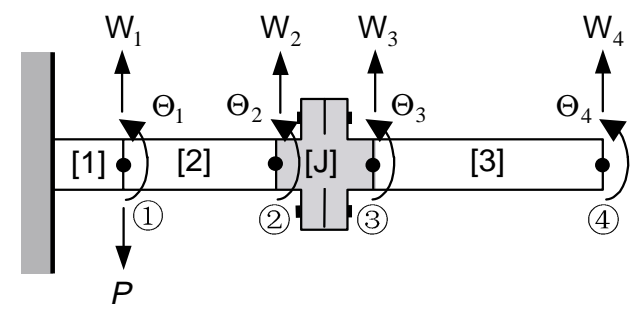

Fig. 4. Spectral element modeling for the beam structure with a bolt-joint.

if there are structural discontinuities along a structural element due to the change of geometric or material properties, the structural element should be further discretized into more spectral elements. The present beam structure has a bolt-joint and an externally applied exciting force $p(t)$. Thus, as shown in Fig. 4, the beam structure should be so divided into four finite elements that it now consists of three uniform Timoshenko-beam elements and one bolt-joint element that is indicated by the symbol $[J]$. As shown in Fig. 4, it is assumed that the external force is applied at node 1.

A single spectral Timoshenko-beam element has two nodal DOF at each end node. Thus, there are total eight nodal DOF to be determined. The eight nodal DOF can be classified into two groups: one group is for the nodal DOF that can be readily measured by experiments (simply, measurable DOF) and the other group is for the nodal DOF that cannot be measured with ease (simply, unmeasurable DOF). In general, it is relatively easier to measure the transverse nodal DOF than to measure the rotational nodal DOF. Thus, in the present study, the transverse nodal DOF are considered as the measurable DOF, $d_{m}$, and the rotational nodal DOF as the unmeasurable DOF, $d_{u}$ :

$$
\begin{aligned}
& \boldsymbol{d}_{m}=\left\{\begin{array}{llll}
W_{1} & W_{2} & W_{3} & W_{4}
\end{array}\right\}^{T} \\
& \boldsymbol{d}_{u}=\left\{\Theta_{1} \Theta_{2} \Theta_{3} \Theta_{4}\right\}^{T}
\end{aligned}
$$

There are total eight unknowns including four rotational nodal DOF (i.e., $\Theta_{1}, \Theta_{2}, \Theta_{3}$, and $\Theta_{4}$ ) and four bolt-joint parameters (i.e., $m, I, k_{v}$, and $k_{t}$ ). Thus, one may need total eight equations to uniquely determine the eight unknowns. This requirement can be met by discretizing the beam structure into four spectral elements as shown in Fig. 4. Adding one more spectral element increases the number of equation by two while the number of unknowns by one. However, if there are more unknowns for any reasons, the additional equations required to make a well-posed problem can be obtained by adding more spectral elements. 
The beam structure consists of four spectral elements, with one clamped boundary at the root of beam. Thus, assembling all spectral elements into a global system, applying the clamped boundary conditions to the result, and lastly rearranging the nodal DOF and forces, the ( 8 by 8 ) global system matrix equation can be derived in the form

$$
\left[\begin{array}{ll}
\boldsymbol{K}_{o m} & \boldsymbol{K}_{o u} \\
\boldsymbol{K}_{\text {em }} & \boldsymbol{K}_{e u}
\end{array}\right]\left\{\begin{array}{l}
\boldsymbol{d}_{m} \\
\boldsymbol{d}_{u}
\end{array}\right\}=\left\{\begin{array}{l}
\boldsymbol{f}_{o} \\
\boldsymbol{f}_{e}
\end{array}\right\}
$$

where

$$
\begin{aligned}
& \boldsymbol{f}_{o}=\left\{\begin{array}{llll}
0 & 0 & 0 & 0
\end{array}\right\}^{T} \\
& \boldsymbol{f}_{e}=\left\{\begin{array}{lllll}
P & 0 & 0 & 0
\end{array}\right\}^{T}
\end{aligned}
$$$$
\boldsymbol{K}_{\text {om }}=
$$

$$
\left[\begin{array}{cccc}
s_{31}^{2} & s_{33}^{2}+k_{v} & -k_{v} & 0 \\
s_{41}^{2} & s_{43}^{2} & 0 & 0 \\
0 & -k_{v} & k_{v}-m \omega^{2}+s_{11}^{3} & s_{13}^{3} \\
0 & 0 & s_{21}^{3} & s_{23}^{3}
\end{array}\right]
$$$$
\boldsymbol{K}_{\text {ou }}=
$$

$$
\begin{aligned}
& {\left[\begin{array}{ccccc}
s_{32}^{2} & s_{34}^{2} & & 0 & 0 \\
s_{42}^{2} & s_{44}^{2}-k_{t} & k_{t} & 0 \\
0 & 0 & & s_{12}^{3} & s_{14}^{3} \\
0 & k_{t} & I \omega^{2}-k_{t}+s_{22}^{3} & s_{24}^{3}
\end{array}\right] } \\
\boldsymbol{K}_{\text {em }}= & {\left[\begin{array}{cccc}
s_{33}^{1}+s_{11}^{2} & s_{13}^{2} & 0 & 0 \\
s_{43}^{1}+s_{21}^{2} & s_{23}^{2} & 0 & \\
0 & 0 & s_{31}^{3} & s_{33}^{3} \\
0 & 0 & s_{41}^{3} & s_{43}^{3}
\end{array}\right] } \\
\boldsymbol{K}_{e u}= & {\left[\begin{array}{cccc}
s_{34}^{1}+s_{12}^{2} & s_{14}^{2} & 0 & 0 \\
s_{44}^{1}+s_{22}^{2} & s_{24}^{2} & 0 & \\
0 & 0 & s_{32}^{3} & s_{34}^{3} \\
0 & 0 & s_{42}^{3} & s_{44}^{3}
\end{array}\right] }
\end{aligned}
$$

In Eq. (15b), $P$ is the Fourier spectrum of the exciting force $p(t)$. The superscripts used in the matrices of Eq. (15) indicate the spectral element numbers specified in Fig. 4, and the subscripts indicate the components of spectral element matrix.

It is very common to use the accelerometers to measure the vibration responses because it is easy-to-apply and cheap. Thus, it seems to be more convenient to express Eq. (14) in terms of the nodal inertance FRF [10], instead of the nodal DOF, as

$$
\left[\begin{array}{ll}
\boldsymbol{K}_{o m} & \boldsymbol{K}_{o u} \\
\boldsymbol{K}_{e m} & \boldsymbol{K}_{e u}
\end{array}\right]\left\{\begin{array}{l}
\boldsymbol{D}_{m} \\
\boldsymbol{D}_{u}
\end{array}\right\}=\left\{\begin{array}{l}
\boldsymbol{F}_{o} \\
\boldsymbol{F}_{e}
\end{array}\right\}
$$

where

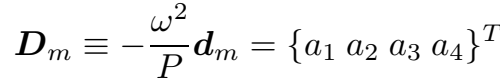

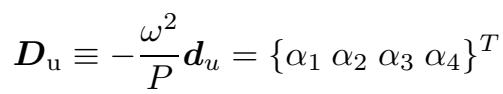

$$
\begin{aligned}
& \boldsymbol{F}_{o} \equiv-\frac{\omega^{2}}{P} \boldsymbol{f}_{o}=\left\{\begin{array}{llll}
0 & 0 & 0 & 0
\end{array}\right\}^{T} \\
& \boldsymbol{F} \equiv-\frac{\omega^{2}}{P} \boldsymbol{f}_{e}=\left\{\begin{array}{llll}
\omega^{2} & 0 & 0 & 0
\end{array}\right\}^{T}
\end{aligned}
$$

In above, $a_{i}$ and $\alpha_{i}$ represent the nodal transverse inertance FRF and the nodal rotational inertance FRF, respectively.

Since the force vector $\boldsymbol{F}_{o}$ is zero, Eq. (16) can be solved for the unmeasurable DOF:

$$
\boldsymbol{D}_{\mathrm{u}}=\boldsymbol{K}_{e u}^{-1}\left[\boldsymbol{F}_{e}-\boldsymbol{K}_{e m} \boldsymbol{D}_{\mathrm{m}}\right]
$$

Equation (18) shows that the unmeasurable (nodal) inertance FRF $\left(\boldsymbol{D}_{u}\right)$ can be computed from the exciting force $\boldsymbol{F}_{e}$ and the measurable (nodal) inertance FRF $\left(\boldsymbol{D}_{m}\right)$, all known in advance. From the first low of Eq. (16), the bolt-joint parameters can be expressed in terms of the unmeasurable inertances as

$$
\begin{aligned}
k_{v}= & \left(s_{31}^{2} a_{1}+s_{32}^{2} \alpha_{1}+s_{33}^{2} a_{2}+s_{34}^{2} \alpha_{2}\right) / \\
& \left(a_{3}-a_{2}\right) \\
k_{t}= & \left(s_{41}^{2} a_{1}+s_{42}^{2} \alpha_{1}+s_{43}^{2} a_{2}+s_{44}^{2} \alpha_{2}\right) / \\
& \left(\alpha_{2}-\alpha_{3}\right) \\
m=\lfloor & \left\lfloor k_{v}\left(a_{3}-a_{2}\right)+s_{11}^{3} a_{3}+s_{12}^{3} \alpha_{3}\right. \\
& \left.+s_{13}^{3} a_{4}+s_{14}^{3} \alpha_{4}\right\rfloor /\left(\omega^{2} a_{3}\right) \\
I= & k_{t}\left(\alpha_{3}-\alpha_{2}\right)-s_{21}^{3} a_{3}-s_{22}^{3} \alpha_{3} \\
& \left.-s_{23}^{3} a_{4}-s_{24}^{3} \alpha_{4}\right\rfloor /\left(\omega^{2} \alpha_{3}\right)
\end{aligned}
$$

Substituting the unmeasurable inertances, all predicted from Eq. (18), into Eq. (19) makes it possible to predict the values of bolt-joint parameters. Once the dynamic properties of a bolt-joint are identified in terms of four equivalent parameters (i.e., the mass, mass moment of inertia, transverse spring, and the torsional spring), the vibration analysis for the beam structure can be readily accomplished by using the wellestablished spectral element analysis procedure [4,6], which is conceptually illustrated in Fig. 5. The numerically clamped condition considered herein to derive Eq. (14) may not be exact and thus should have some influence on the estimated joint parameters. To identify the dynamic characteristics of real beam boundary conditions, one may refer to the previous work by the author [11]. 


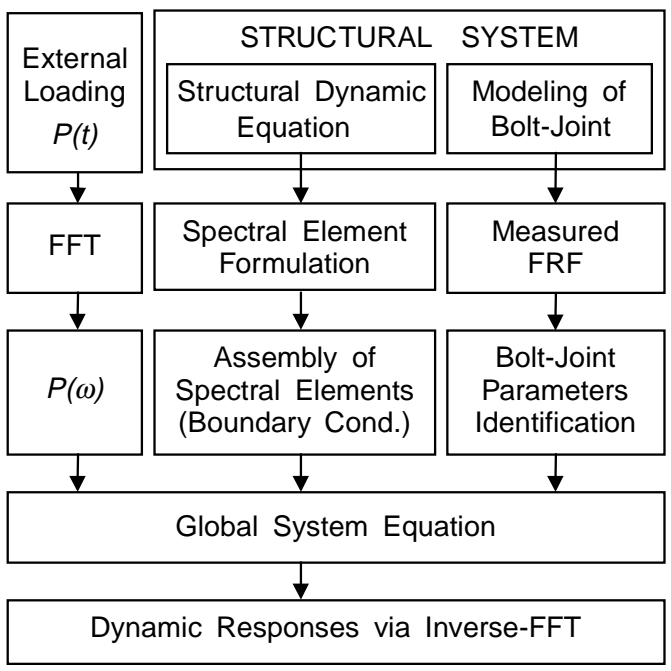

Fig. 5. Spectral element analysis procedure for the beam structure with a bolt-joint.

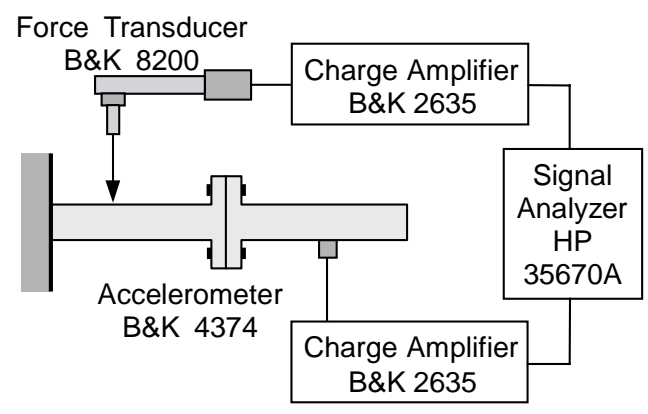

Fig. 6. Schematic of the experimental setup.

\section{Experiment, analysis, and discusion}

\subsection{Experiment}

Experiments are conducted for an aluminum beam structure with a single bolt-joint. The experimental setup is shown in Fig. 6. Two same length of uniform beams are connected at the mid-point by the bolt-joint The total length of beam structure is $0.56 \mathrm{~m}$, and the width and thickness are $0.03 \mathrm{~m}$ and $0.01 \mathrm{~m}$, respectively.

The vise is carefully designed so as to minimize its effects on the dynamic characteristics of beam specimen within the frequency range of concern, i.e. $1.2 \mathrm{kHz}$. Impact hammer is used to apply wide exciting spectrum. The impact point and the response measurement point are chosen not to be near the node points (points of zero motion) [10]. The coherence functions are carefully reviewed to select the final response measurement points. In the signal analyzer, the sampling time is setup as 1 second. Although the vibration test for the simple beams is not a hard task in these days, the same test has been repeated many times, and the best test results are chosen for use. The frequency range of concern in this paper is about $1.2 \mathrm{kHz}$, for both theoretical analysis and experiments. The lowest natural frequency for the axial vibration of the present beam structure is found to be about $2.3 \mathrm{kHz}$, which is much higher than the frequency range of concern in this paper. Thus, the effects of axial modes are neglected in the preceding theoretical formulation and also in the following analytical prediction of vibration responses. Measured exciting forces and accelerations are transformed into the frequency domain by using the FFT algorithm of MATLAB ${ }^{\circledR}$, and the results are used to compute the nodal rotational inertances defined in Eq. (18). Figure 7 shows the FRF (nodal transverse inertances) measured at nodes $1,2,3$, and 4 .

\subsection{Analysis and discussion}

Using the measured FRF shown in Fig. 7, first the unmeasurable nodal rotational inertances are predicted from Eq. (18). Figure 8 shows the predicted rotational inertances at each node. The predicted rotational inertances are then used to predict the four parameters of equivalent bolt-joint model from Eq. (19). Figures 9 and 10 shows the predicted inertia and elastic properties (i.e., transverse spring constant, torsional spring constant, mass, and mass moment of inertia) of the equivalent bolt-joint model. They are all found to be frequency-dependent. In the preceding section, it is shown that the spectral element matrix for a structural element is in general frequency-dependent. This is why the spectral element matrix is often called the dynamic stiffness matrix in the literature [7]. By using the predicted inertia and elastic properties shown in Figs 9 and 10, the spectral element matrix for the equivalent bolt-joint model can be computed from Eq. (12). As the word 'spectral element matrix' implies, it is natural that the predicted inertia and elastic properties are all frequency-independent. Thus, the readers should not be in confusion because of the terminology 'spring constant' that is widely used in the literature to represent the elastic stiffness of a structure.

To evaluate the accuracy of the equivalent boltjoint model, the experimentally measured vibration responses are compared with analytically predicted ones. The SEM is used for the analytically predicted vibration responses. The spectral element matrix for the bolt-joint element is computed from Eq. (12) by using 


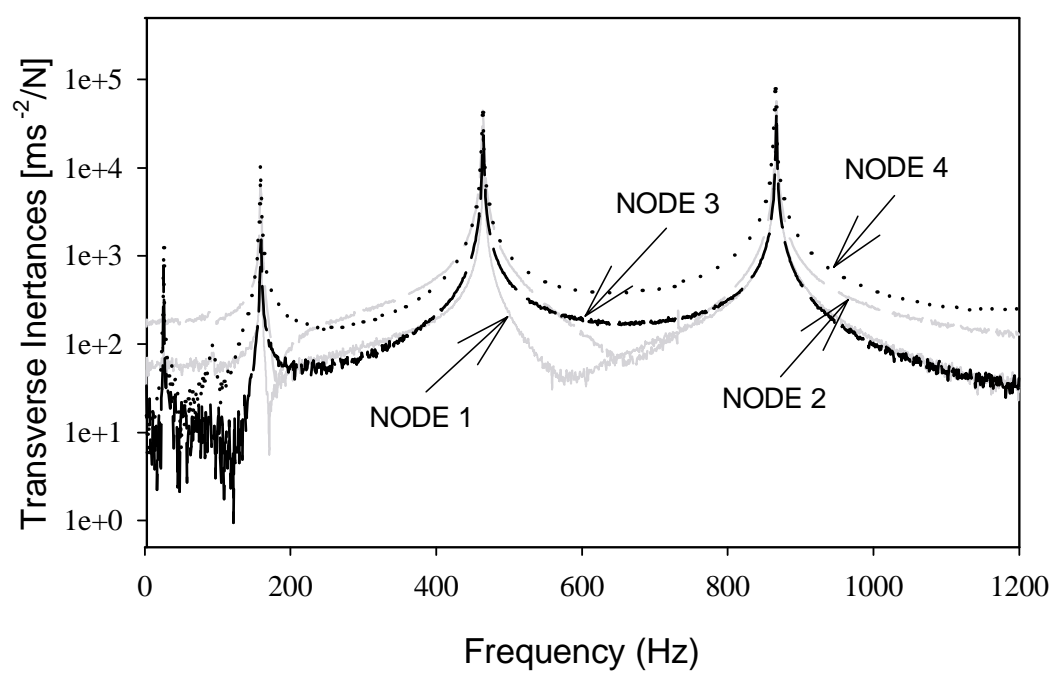

Fig. 7. Measured transverse inertance FRF at nodes $1-4$ of the cantilevered beam structure with a bolt-joint.

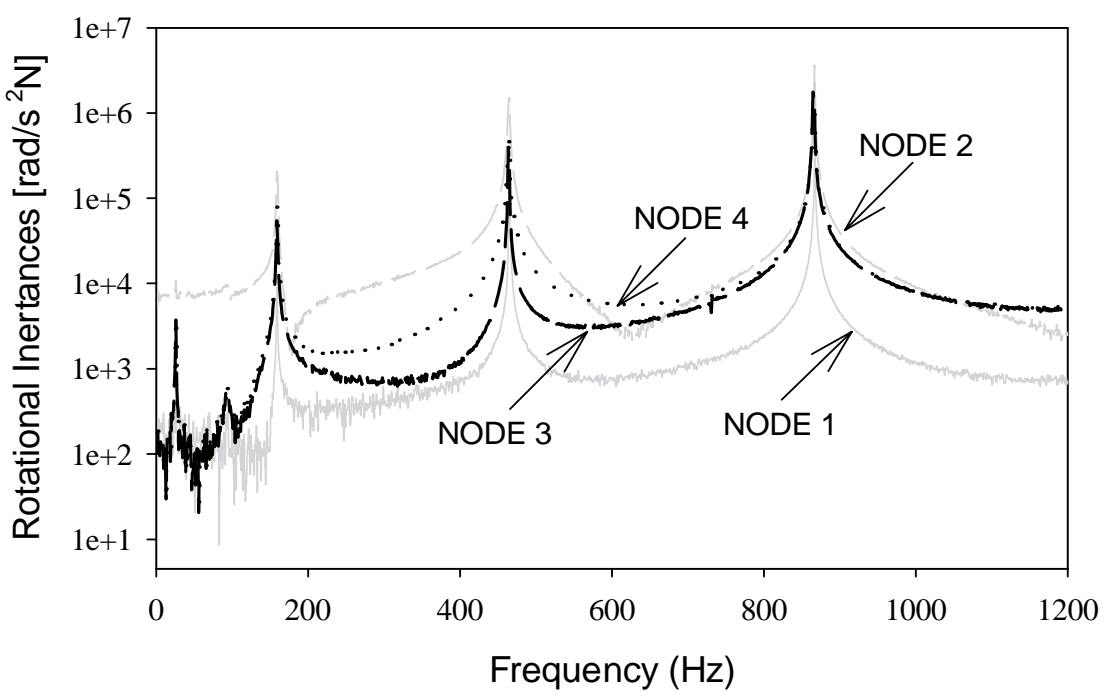

Fig. 8. Predicted rotational inertance FRF at nodes $1-4$ of the cantilevered beam structure with a bolt-joint.

the four bolt-joint parameters, all predicted from the measured FRF. The spectral element matrices for the bolt-joint element and two Timoshenko-beam elements are then assembled in a completely analogous way that used for FEM. Finally, by applying the clamped boundary conditions at the root of beam structure to the result of assembly, the global system equation can be obtained to predict the vibration responses by SEM [4,6]. The spectral element analysis procedure used in this paper is conceptually illustrated in Fig. 5.

The analytically predicted and measured transverse inertances at the free-end node 4 are compared in Fig. 12. It is shown that these two frequency-domain results are extremely well matched to each other. Similarly, the analytically predicted and measured accelerations at the free-end node 4 are compared in Fig. 13. The two time-domain results are shown to be very close to each other, but now with small discrepancy. Since the predicted acceleration is computed from the predicted FRF by using the inverse-FFT algorithm, the small discrepancy is mostly due to the miss of high frequency spectral components beyond the Nyquist frequency [12] and also due to the inherent computation error. One should note that the high frequency spectral components beyond Nyquist frequency could not be included in the time-domain solutions because the 


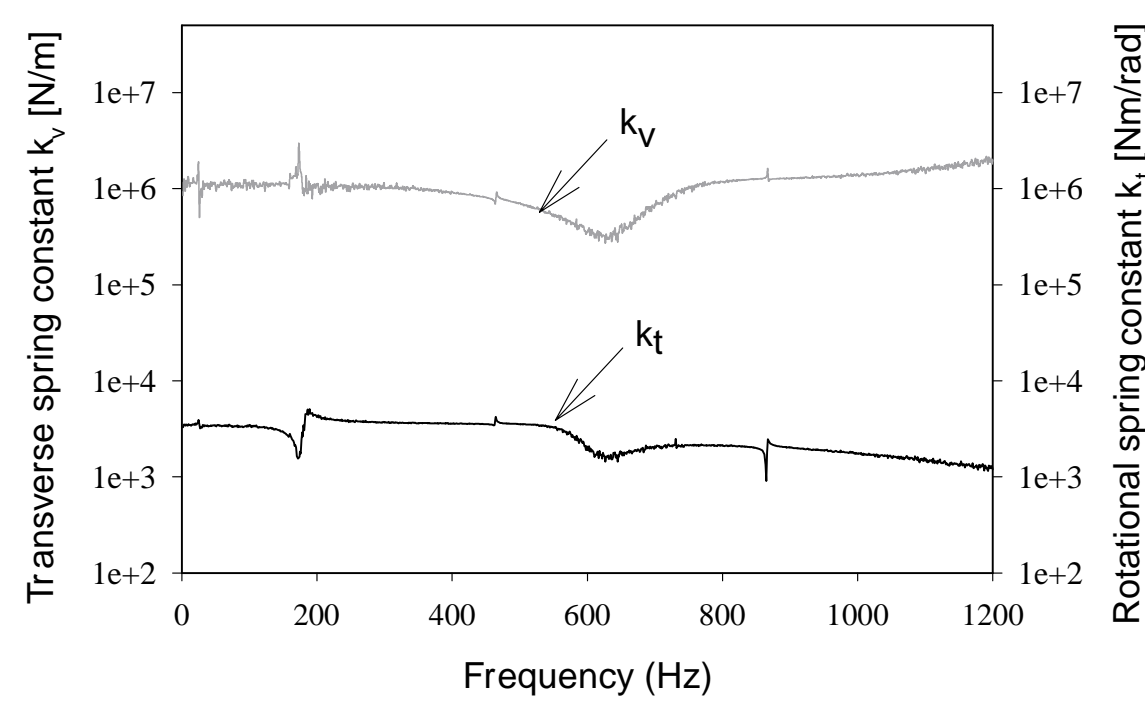

Fig. 9. Predicted equivalent transverse and torsional spring constants for the equivalent bolt-joint model.

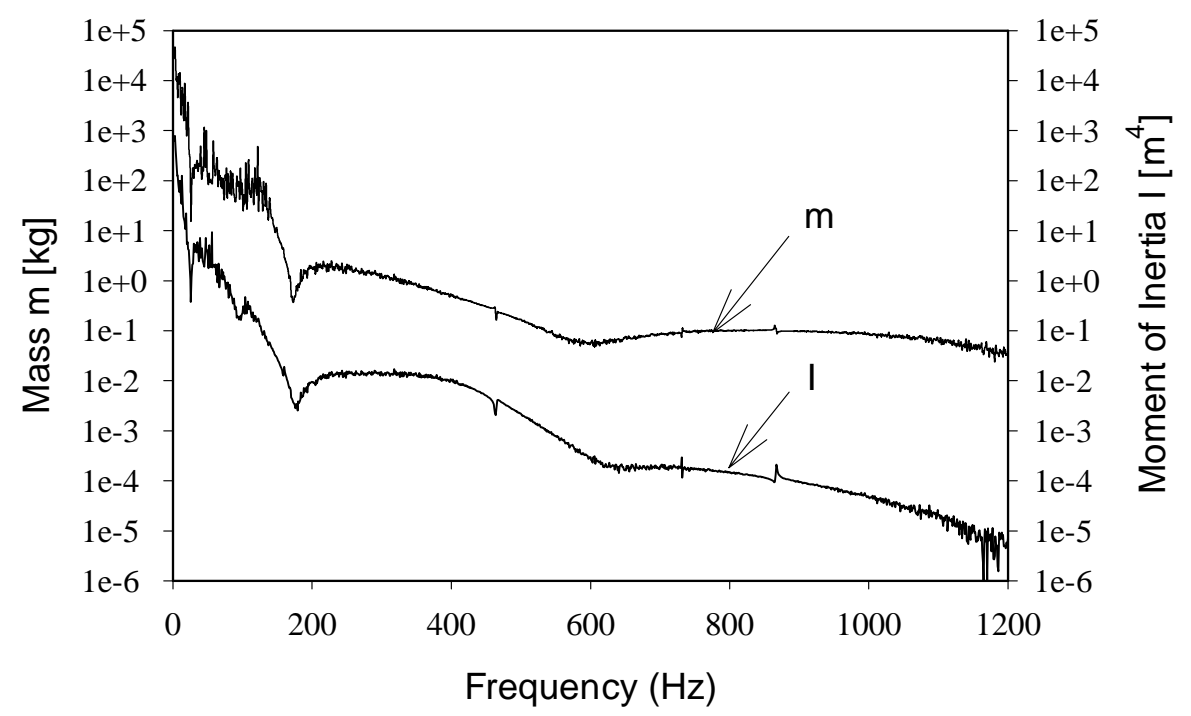

Fig. 10. Predicted equivalent mass and the mass moment of inertia for the equivalent bolt-joint model.

forward- and inverse-FFT algorithms are used in the spectral element analysis procedure [4]. The analytical predictions can be further improved by choosing the best values of the time window and Nyquist frequency [4].

\section{Conclusions}

This paper introduces a method to identify the dynamic characteristics of the bolt-joint within a beam structure. A cantilevered beam structure with a bolt- joint is considered as an illustrative problem. The bolt-joint part is represented by the equivalent spectral element with four parameters and the rest part of the beam structure is represented by the spectral Timoshenko-beam elements. The dynamic characteristics of the bolt-joint are identified by determining the four bolt-joint parameters from the experimentally measured FRF. To verify the validity of the present SEM/FRF-based identification method, the vibration responses measured by experiments are compared with those analytically predicted by using the identified boltjoint parameters. It is shown that the analytical pre- 


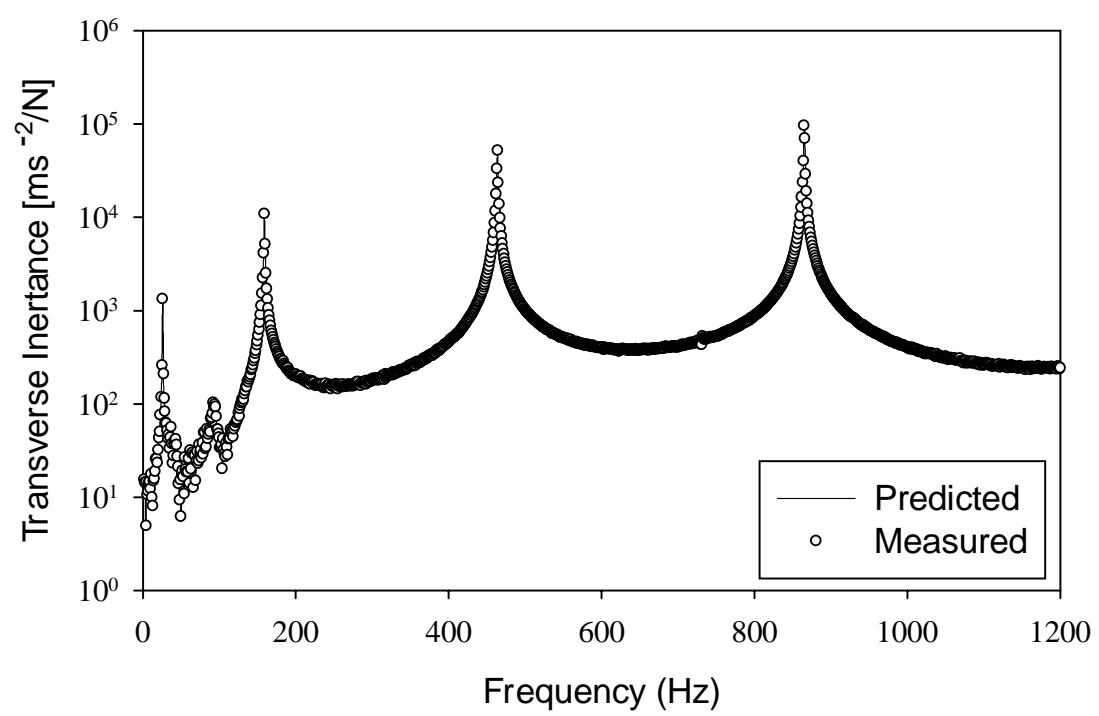

Fig. 11. Comparison of the measured and predicted transverse inertance FRF at node 4 of the cantilevered beam with a bolt joint.

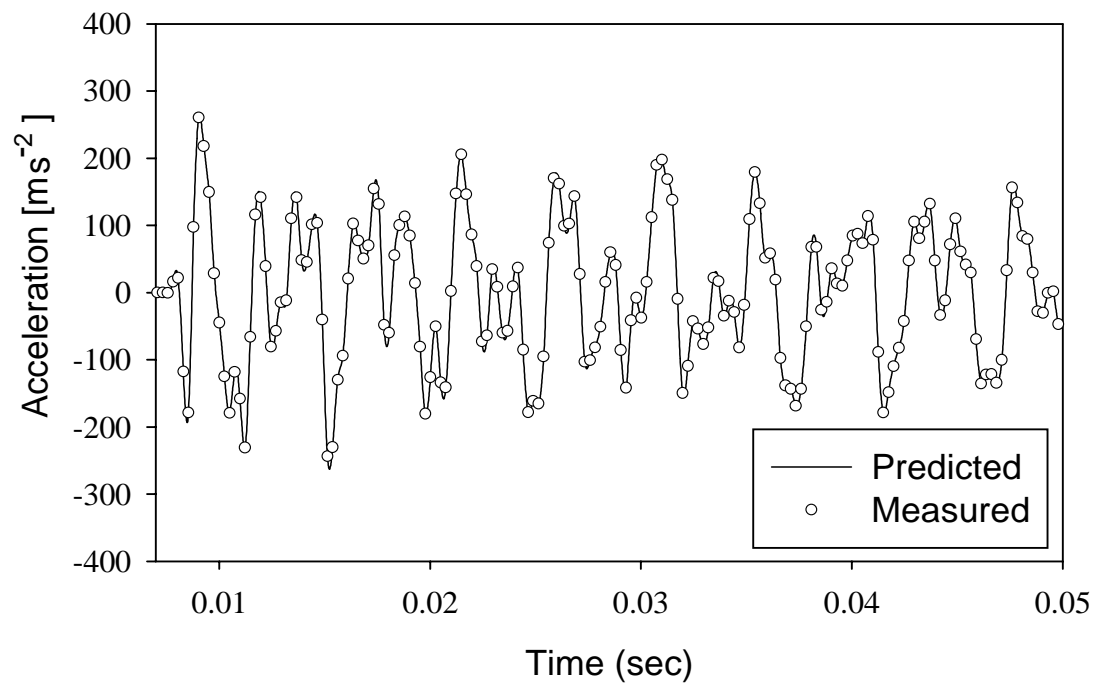

Fig. 12. Comparison of the measured and predicted accelerations at node 4 of the cantilevered beam with a bolt joint.

dictions are in general very close to the experimentally measured results.

\section{References}

[1] J.X. Yuan and X.M. Wu, Identification of joint structural parameters of machine tool by DDS and FEM, Journal of Engineering for Industry 107 (1985), 64-69.

[2] J.S. Tsai and Y.F. Chou, The identification of dynamic characteristics of a single bolt joint, Journal of Sound and Vibration 125(3) (1988), 487-502.

[3] A.S. Nobari, D.A. Robb and D.J. Ewins, Model updating and joint identification methods, restrictions and overlap, Interna- tional Journal of Analytical and Experimental Modal Analysis V8 (1933), 95-103.

[4] J.F. Doyle, Wave propagation in structures, (2nd ed.), Springer-Verlag, New York, 1997.

[5] U. Lee and J. Lee, Spectral-element method for levy-type plates subject to dynamic loads, Journal of Engineering Mechanics 125(2) (1999), 243-247.

[6] U. Lee, J. Kim and A.Y.T. Leung, Spectral element method in structural dynamics, The Shock and Vibration Digest 32(6) (2000), 451-465.

[7] J.R. Banerjee, Dynamic stiffness formulation for structural elements: a general approach, Computers and Structures 63 (1997), 101-103.

[8] S.K. Clark, Dynamics of continuous elements, Prentice-Hall, Englewood Cliffs, New Jersey, 1972. 
[9] E.C. Pestel and F.A. Leckie, Matrix methods in elastomechanics, McGraw-Hill, New York, 1963.

[10] D.J. Ewins, Modal testing: theory and practice, Research Studies Press, Hertfordshire, England, 1986.

[11] U. Lee and J. Kim, Determination of non-ideal beam boundary conditions: a spectral element approach, AIAA Journal 38(2) (2000), 309-316.

[12] E.O. Brigham, The fast Fourier transform and its applications, Prentice-Hall, London, England, 1988. 

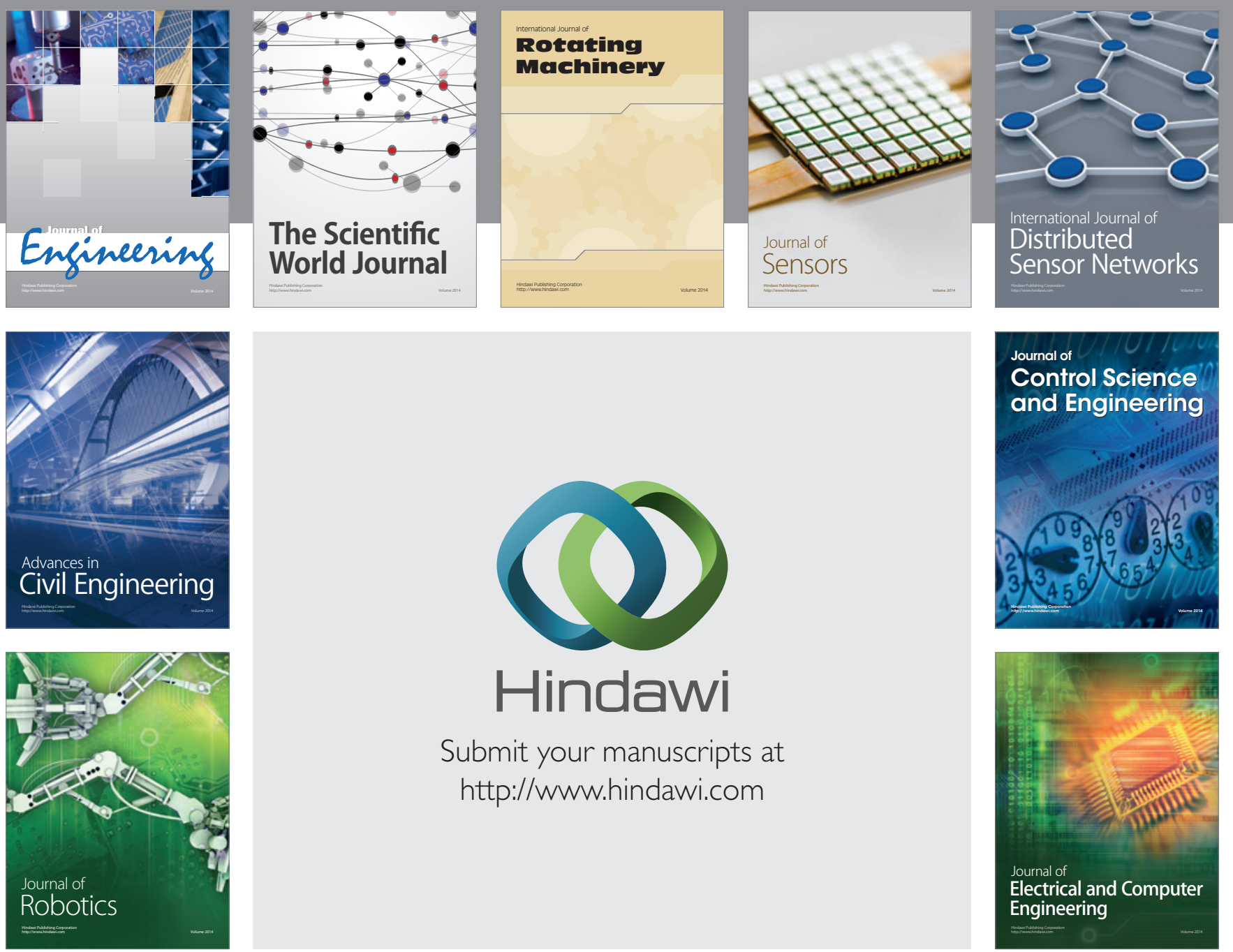

Submit your manuscripts at

http://www.hindawi.com
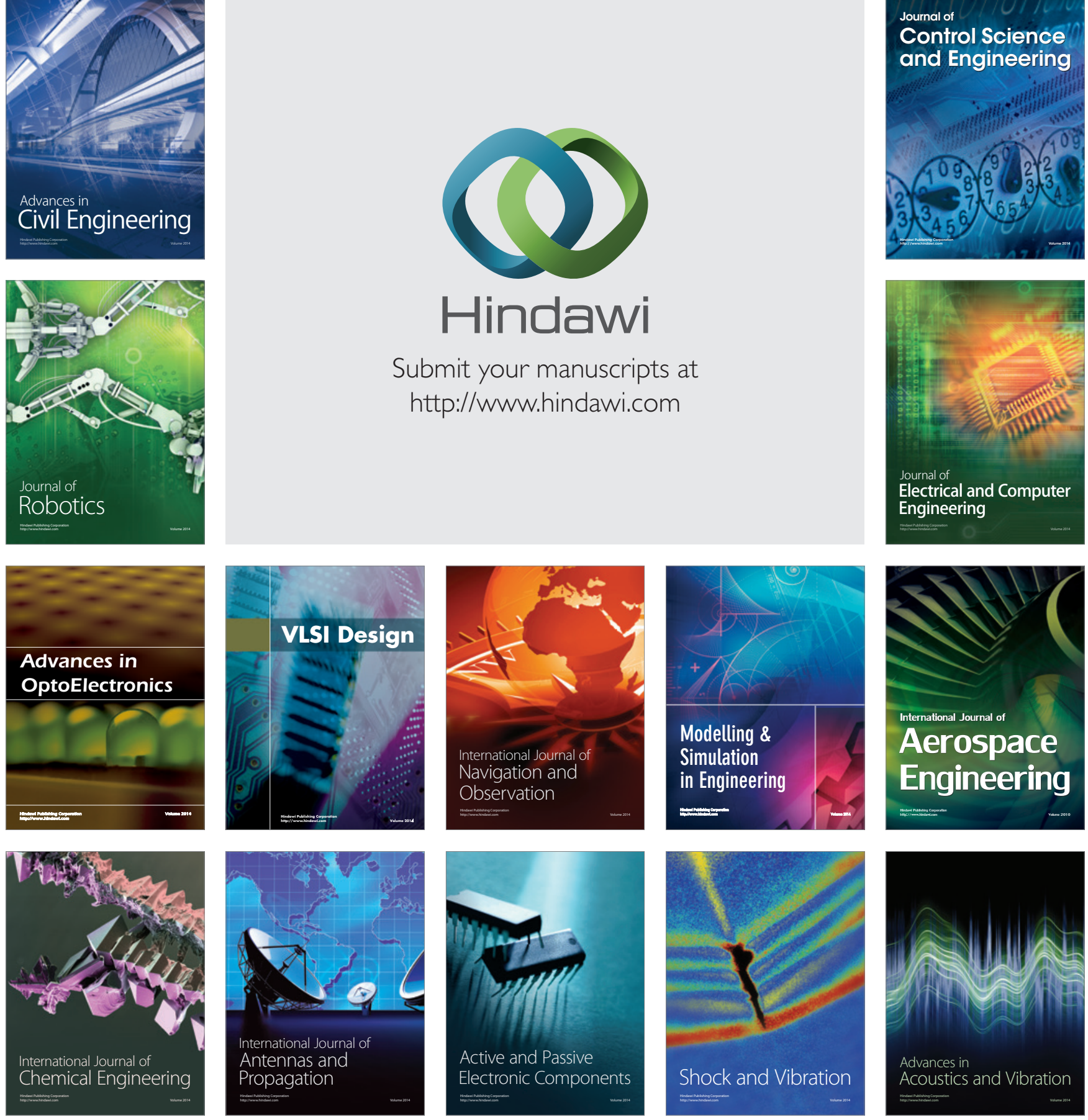